\title{
Démarche qualité en médecine: un état des lieux par portraits succincts (6)
}

* www fmh.ch $\rightarrow$ Welcome $\rightarrow$ Qui sommes-nous? $\rightarrow$ Le Secrétariat général $\rightarrow$ Données, démographie \& qualité.
Dans notre pays, la démarche qualité en médecine est en plein développement et s'est établie dans de nombreux endroits. C'est ce que le groupe de travail Qualité de la FMH (GTQ-FMH) tient à montrer aux membres de la FMH. Il présente ainsi dans cinq numéros consécutifs du BMS les initiatives Qualité qui lui sont annoncées depuis 2006 par des médecins de toutes les disciplines médicales et de tous les secteurs d'activité (domaine hospitalier/ambulatoire). Ces descriptifs sont également publiés sur le site Internet de la FMH / Département Données, démographie et qualité avec des informations pratiques telles que graphiques ou bibliographie. * La deuxième série de descriptifs sur les démarches qualité commence par la norme ISO 9001:2008 et la méthode appliquée par la Communauté de travail pour l'assurance-qualité en chirurgie.

\section{Norme DIN EN ISO 9001:2008}

Correspondance: Dr Rafael E. Sinniger MBA HSG

SQS-Association suisse pour systèmes de qualité et de management Bernstrasse 103

Case postale 686 CH-3052 Zollikofen Tél. 0319103535 Fax 0319103545

headoffice@sqs.ch

\section{Description}

La norme DIN EN ISO 9001:2008

- est reconnue dans le monde entier pour certifier des systèmes de gestion de la qualité;

- fixe des exigences sans prescrire de solutions;

- met l'accent sur des audits internes et externes.

Les normes ISO sont des standards reconnus sur le plan international pour la certification de systèmes de gestion de qualité. Elles visent la satisfaction des clients, la maîtrise des processus et l'amélioration continue. La norme DIN EN ISO 9001:2008, par exemple, porte sur le management de la qualité, la responsabilité de la direction, la gestion des ressources, la réalisation de produits ainsi que la mesure, l'analyse et l'amélioration des prestations. L'ISO (organisation internationale de normalisation) fonctionne d'après la formule «planifier, agir, contrôler, améliorer». Des audits internes et externes sont menés avant la certification, laquelle est valable trois ans. Des audits de surveillance sont effectués chaque année durant cette période.

En Suisse, la certification ISO est réalisée avant tout dans le domaine hospitalier. Elle peut toutefois être adaptée avec souplesse aux besoins des cabinets médicaux: il existe une grande marge de manœuvre dans l'élaboration des normes. Jusqu'à présent, le nombre de cabinets médicaux certifiés ISO est peu élevé, ce qui rend plus difficile un benchmarking ou un échange. Il existe néanmoins différentes entreprises qui soutiennent les cabinets médicaux sur la voie de la certification (cf. informations supplémentaires).

\section{Applicabilité}

Entreprises comptant une à plusieurs milliers de personnes

\section{Charges et coûts}

Conformément aux indications du service d'accréditation:

- certification de la plus petite unité (1 à 4 postes à plein temps): env. 4000 francs puis env. 1500 francs par année pour les mesures visant à maintenir le certificat;

- les coûts sont adaptés au cabinet médical ou à la clinique, c'est-à-dire à sa taille et/ou à sa complexité;

- durant la phase de mise en place, pendant quelques mois, les charges peuvent atteindre $10 \%$ de la prestation de chaque collaborateur.

\section{Informations supplémentaires}

www.sqs.ch (Association suisse pour systèmes de qualité et de management, chargée de la réalisation des certifications ISO) 


\section{Communauté de travail pour I'assurance de la qualité en chirurgie (AQC)}

Luzi Rageth

Correspondance:

Dr oec. HSG Luzi Rageth, MA CQC Communauté de travail pour l'assurance-qualité en chirurgie Secrétariat

Nussgasse 5

$\mathrm{CH}-8008$ Zurich

Tél. 0444452667

Fax 0443802274

Hotline 0794009932

aqc@aqc.ch

\author{
Description \\ La Communauté de travail pour I'assurance \\ de la qualité en chirurgie (AQC) \\ - documente la démarche-qualité dans une \\ banque de données pour tous les cas \\ médicaux (opérations, enfants, résultats); \\ - dispose d'un outil d'évaluation complet \\ et des possibilités du benchmarking; \\ - est certifiée ISO.
}

L'AQC est un projet officiel des Sociétés suisses de chirurgie (SSC), de chirurgie de la main (SSCM), de neurochirurgie (SSNC) et de chirurgie viscérale (SSCV) comme de l'Association Suisse des médecins avec activité chirurgicale et invasive (fmCh). Elle se caractérise par une documentation prospective sur la qualité relative à tous les cas médicaux (opérations, enfants, résultats; actuellement 450000 cas). Comme ces données sont recensées depuis 13 ans, elles permettent aujourd'hui de faire des pronostics différenciés au sujet des complications.

Les données de l'AQC sont basées sur une autodéclaration. Le benchmark est effectué entre les cliniques participantes et l'échantillonnage complet ou le groupe de pairs caractérisé par une qualité de données particulièrement bonne. La plus haute priorité est accordée à la protection des données. Le nombre de complications saisies dans la banque de données s'accroît régulièrement en raison de l'augmentation de la confiance dans le système et, partant, de la déclaration plus fréquente de ces cas. Il serait possible et judicieux d'élargir le système AQC au domaine non opérationnel moyennant quelques modifications.

L'utilité d'un processus AQC pour la qualité médicale réside dans la promotion de la transparence interne et les répercussions de cette transparence vers l'extérieur. Certifiée ISO, l'AQC est un élément important d'une gestion de la qualité qui se veut complète.

\section{Applicabilité}

L'AQC est intéressante avant tout pour les cliniques. Près d'un tiers des hôpitaux de formation postgraduée participent à une AQC.

\section{Charges et coûts}

15 min au maximum suffisent pour remplir un questionnaire; un questionnaire réduit est à la disposition des médecins agréés. L'AQC prend en charge la saisie et l'évaluation des données; elle remet deux fois par année des évaluations standards aux cliniques membres.

Les coûts de saisie des données sont bas pour un médecin / une clinique (Fr. 1.50 par cas pour la livraison électronique des données et env. Fr. 6.-l cas pour la saisie sur papier). Il n'y a pas d'autres coûts initiaux ou fixes supplémentaires.

\section{Informations supplémentaires}

www.aqc.ch 